\title{
VACUUM ENERGY DENSITY AND PRESSURE NEAR BOUNDARIES
}

\author{
S. A. FULLING* \\ Mathematics Department, Texas A\&M University, \\ College Station, TX 77843-3368, USA \\ *E-mail: fulling@math.tamu.edu \\ www.math.tamu.edu/ fulling/
}

\begin{abstract}
In vacuum energy calculations with an ultraviolet cutoff, divergences arise that clearly are related to the physics of boundaries. We point out how to find the cut-off energy density and pressure most directly from a Green function. Then we discuss three apparent paradoxes arising in this work that are in various stages of resolution.

Keywords: Vacuum energy; Stress tensor; Cylinder kernel; Optical approximation; Multiple scattering.
\end{abstract}

\section{Introduction}

The main principles of our working philosophy toward vacuum energy and its divergences are the following. ${ }^{1}$

Local energy density and pressure, the components of the stress tensor $T^{\mu \nu}(\mathbf{r})$, are important for understanding the physics. Semiclassical path analysis (reducing in the simplest cases to exact image solutions) is illuminating. Idealized models with perfect reflection boundary conditions (and a scalar field, often in lower dimensions) can still be instructive, provided one is honest and sensible about their limitations.

To understand the physics of divergences, it is best to use an ultraviolet cutoff. Zeta and dimensional methods of regularization hide the divergences in an ad hoc way and yield global energies that are inconsistent with the local $T^{\mu \nu}(\mathbf{r})$ and hence with general relativity. ${ }^{2}$ At the other extreme, detailed modeling of real materials is hard, and also too specific: real metals have little to do with hadron bags, cosmological branes, thermal fluctuations in biological membranes, and other situations where Casimir-type calculations have been conducted. An ultraviolet cutoff in quantum vacuum 
calculations is like the repulsive core in the Lennard-Jones potential. A more accurate atomic potential would be based on the electronic structure of atoms - but would not apply to nucleon-nucleon scattering. Similarly, a simple frequency cutoff is universally applicable, mathematically convenient, and qualitatively physically plausible — but can't be expected to model real metals at atomic-scale distances.

Our mathematically preferred cutoff is the factor $e^{-t \omega_{j}}$, but others are possible and may be physically superior. (Note that the $\omega_{j}^{-2}$ of dielectric theory is not sufficient. Further account of the granular structure of matter is needed. ${ }^{3,4}$ ) Thus we study a regularized total energy

$$
E(t) \equiv \frac{1}{2} \sum_{j=1}^{\infty} \omega_{j} e^{-t \omega_{j}} \quad(t \downarrow 0)
$$

and a regularized energy density

$$
\rho(\mathbf{r}, t) \equiv\left\langle T^{00}\right\rangle \equiv \frac{1}{2} \sum_{j=1}^{\infty} \omega_{j}\left|\phi_{j}(\mathbf{r})\right|^{2} e^{-t \omega_{j}}
$$

with similar formulas for the other components of $T^{\mu \nu}$. (Equation (2) applies to the value $\xi=\frac{1}{4}$ for the curvature coupling parameter. Other cases are similar but more complicated. ${ }^{1}$ )

\section{Integral Kernels in Billiards and Cavities}

Associated with any self-adjoint second-order linear differential operator $H$ is a dizzying array of integral kernels (also called Green functions or spectral functions), most of which implement the solution of some partial differential equation involving $H$. They include the resolvent kernel (energydomain Green function) $G\left(k, \mathbf{r}, \mathbf{r}^{\prime}\right)=\left\langle\mathbf{r}\left|\left(H-k^{2}\right)^{-1}\right| \mathbf{r}^{\prime}\right\rangle$, the heat kernel $K\left(t, \mathbf{r}, \mathbf{r}^{\prime}\right)=\left\langle\mathbf{r}\left|e^{-t H}\right| \mathbf{r}^{\prime}\right\rangle$, the quantum kernel $U\left(t, \mathbf{r}, \mathbf{r}^{\prime}\right)=\left\langle\mathbf{r}\left|e^{-i t H}\right| \mathbf{r}^{\prime}\right\rangle$, the local zeta function $Z\left(s, \mathbf{r}, \mathbf{r}^{\prime}\right)=\left\langle\mathbf{r}\left|H^{-s}\right| \mathbf{r}^{\prime}\right\rangle$, and the cylinder kernel

$$
\bar{T}\left(t, \mathbf{r}, \mathbf{r}^{\prime}\right)=\left\langle\mathbf{r}\left|\frac{e^{-t \sqrt{H}}}{-\sqrt{H}}\right| \mathbf{r}^{\prime}\right\rangle .
$$

Of foremost importance to us is that derivatives of the cylinder kernel comprise the regularized stress tensor:

$$
\rho(\mathbf{r}, t)=-\frac{1}{2} \frac{\partial^{2}}{\partial t^{2}} \bar{T}(t, \mathbf{r}, \mathbf{r}) \quad\left(\text { if } \xi=\frac{1}{4}\right), \quad \text { etc. }
$$

Note that $U(t)=K(i t)$; similarly, the analytic continuation of $\bar{T}$ to imaginary time is one of the Green functions associated with the wave 
equation. In a compact system the eigenvalue density is an integral of the imaginary part of $G$, and this fact has a local counterpart:

$$
\sigma(\mathbf{r}, k) d k=\frac{1}{\pi} \operatorname{Im} G(\mathbf{r}, \mathbf{r}, k) d\left(k^{2}\right) .
$$

A Laplace transform with respect to $k^{2}$ leads from $\sigma$ to $K$, and from $K$ to $G$. A Laplace transform in $k$ leads directly from $\sigma$ to $\bar{T}$, which is also related to $K$ by

$$
\int_{0}^{\infty} t^{-1 / 2} e^{-\tau^{2} / 4 t} K(t) d t=-\sqrt{\pi} \bar{T}(\tau) .
$$

Because of all these interrelations, each spectral function in some sense contains all the information in any of the others, though perhaps in a less (or more) accessible form.

In the asymptotic construction of Green functions it is useful to use what is known in general relativity as the Synge-DeWitt formalism ${ }^{5,6}$ and used in classical optics in different notations. ${ }^{7}$ Let $L\left(\mathbf{r}, \mathbf{r}^{\prime}\right)$ denote the distance from $\mathbf{r}^{\prime}$ to $\mathbf{r}$ along (in the present context) a straight path with specular reflections, and let $\sigma\left(\mathbf{r}, \mathbf{r}^{\prime}\right) \equiv \frac{1}{2} L\left(\mathbf{r}, \mathbf{r}^{\prime}\right)^{2}$. Then $\left(\nabla \equiv \nabla_{\mathbf{r}}\right)$

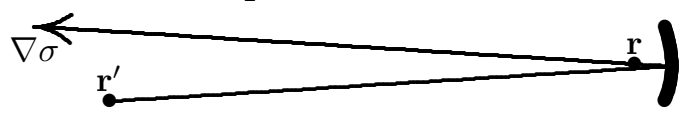

(i) $\nabla \sigma=L \nabla L=L \hat{\mathbf{n}}$, where $\hat{\mathbf{n}}$ is the unit vector at $\mathbf{r}$ in the direction of the path;

(ii) $(\nabla \sigma)^{2}=L^{2}=2 \sigma$;

(iii) $\nabla^{2} \sigma=1+L \nabla \cdot \hat{\mathbf{n}}=d+O\left(L^{2}\right)$ (where $d$ is the spatial dimension, usually 3$)$;

(iv) For the direct path (no reflections), $\sigma=\frac{1}{2}\left|\mathbf{r}-\mathbf{r}^{\prime}\right|^{2}, \nabla \sigma=\mathbf{r}-\mathbf{r}^{\prime}$, and $\nabla^{2} \sigma=d$.

For $H=-\nabla^{2}$ in a cavity, we can now construct any kernel by the optical approximation. Let $G$ stand for the function in question (not necessarily the resolvent kernel). We seek it in the form of a sum over specularly reflecting paths of terms

$$
G_{j}=(-1)^{j} D_{j}\left(\mathbf{r}, \mathbf{r}^{\prime}\right) F\left(\sigma\left(\mathbf{r}, \mathbf{r}^{\prime}\right)\right),
$$

where $(-1)^{j}$ means the parity of the number of reflections (so that the Dirichlet condition is satisfied by the sum), and $D$ does not depend on the parameter $t$ or $k$ (though $F$ does). The central observation of this section is that $D$ comes out the same for all the kernels. (This is true for billiards and cavities, not for Schrödinger operators with potentials, whose kernels 
do not factor as nicely as in Eq. (7).) Thus we have the option of studying $\bar{T}$ directly, instead of starting with $G$ or $U$ and passing through $\sigma$, as usually done.

Proof and construction. Plug ansatz (7) into the partial differential equation defining $G$. Group the terms by order of singularity; it will be seen that the leading term vanishes if $F\left(\frac{1}{2}\left|\mathbf{r}-\mathbf{r}^{\prime}\right|\right)=G_{0}\left(\cdot, \mathbf{r}, \mathbf{r}^{\prime}\right)$, the corresponding kernel in $\mathbf{R}^{3}$. For the most familiar kernels, the singularity occurs in the limit $t \downarrow 0$ or $k \rightarrow+\infty$. For $\bar{T}$, "singularity" refers to powers of $\left(t^{2}+2 \sigma\right)^{-1}$, and the conclusion thus far is that

$$
F=\bar{T}_{0}=-\frac{1}{2 \pi^{2}} \frac{1}{t^{2}+\left|\mathbf{r}-\mathbf{r}^{\prime}\right|^{2}} .
$$

Then the next term vanishes if $D \equiv|\operatorname{det} M|^{1 / 2}$, where $M_{j k} \equiv \partial^{2} \sigma / \partial r_{j} \partial r_{k}^{\prime}$. In other terms, $\operatorname{det} M=L^{d-1} \delta$ where $\delta\left(\mathbf{r}, \mathbf{r}^{\prime}\right)$ is the enlargement factor $d($ angle $) / d$ (area) exploited by Scardicchio and Jaffe. ${ }^{7}$

\section{Paradox Past}

Why is the "renormalized" total energy finite (and independent of $\xi$ ) when the "renormalized" energy density has a nonintegrable, $\xi$-dependent singularity at a boundary? (Here "renormalization" refers to the "unspeakable act" of discarding the divergent terms in $E(t)$ and $\rho(\mathbf{r}, t)$ as $t \rightarrow 0$; this is not the place to discuss its physical justification, or lack thereof.) This question was essentially raised by Deutsch and Candelas ${ }^{2}$ and essentially answered by Ford and Svaiter. ${ }^{8}$ I have dwelt upon it at previous QFExt meetings, so I'll dismiss it quickly here: $\lim _{t \downarrow 0} \rho(\mathbf{r}, t)$ is a limit nonuniform in $\mathbf{r}$, so taking the limit outside the integral over space is not the same as taking it inside. (See figure in Sec. 5.) In fact, the integral $E(t)$ for small, nonzero $t$ may have the same or the opposite sign as $\lim \rho(\mathbf{r}, t)$, depending on $\xi$ and other features of the configuration studied. ${ }^{1}$ When $t$ is finite, so is $E(t)=\iint d S \int \rho d z$ ( $z \equiv$ distance from boundary). Thus the cutoff theory is internally consistent (so far).

\section{Paradox Present}

The cutoff-dependent surface energy of a sphere depends on radius. Where is the corresponding pressure? The regularized energy near a spherical surface of radius $R$, on either the inside or the outside, is

$$
E(t)=-\frac{R^{2}}{2 t^{3}}+O\left(\frac{R}{t^{2}}\right) .
$$


(This result can be derived from either the heat-kernel expansion or the leading term in a multiple-reflection expansion.) It follows that there must be a generalized force and corresponding radial pressure

$$
F=-\frac{\partial E}{\partial R}=\frac{R}{t^{3}}, \quad p_{r}=\frac{F}{4 \pi R^{2}}=\frac{1}{4 \pi R t^{3}} .
$$

For a thin shell these quantities should be multiplied by 2 to account for both inside and outside. (This discussion refers to a scalar field. It is well known that the corresponding quantities vanish for electromagnetism because of cancellation between two classes of modes.)

However, one seeks in vain for such a term in previous direct studies of $T_{\mu \nu}$ near a sphere. ${ }^{9-11}$ Furthermore, in [1] we said that in a rectangle, there is a divergent pressure on a particular side, but it is unrelated to the divergent energy density in the region adjacent to that side (which is independent of the position of the side). Instead, it corresponds to the length dependence of the divergent energy in the edge regions along the perpendicular sides. (In a piston it is cancelled by the exterior.) But in a sphere, there is no perpendicular side! The sphere pressure manifestly comes from $p_{r}$, not the pressure $p_{\perp}$ in the tangential directions, and it must somehow arise from the curvature.

Note that here we are not talking about an aspect of the "first-order curvature term" in the heat kernel, spectral density, and energy. That effect has opposite signs for inside and outside and same sign for Dirichlet and Neumann conditions. This effect must have same sign for inside and outside and opposite signs for Dirichlet and Neumann, like the curvatureindependent surface term in the energy from which it arises.

We first investigate the situation in the optical approximation, using the $\bar{T}$ formalism from above and the enlargement factor $D$ for a sphere calculated by Scardicchio and Jaffe. ${ }^{7}$ With $2 \sigma=4(r-R)^{2}$, we find

$$
\begin{gathered}
\rho=-\frac{2 t^{2} R}{\pi^{2} r}\left(t^{2}+2 \sigma\right)^{-3}+\frac{R}{2 \pi^{2} r}\left(t^{2}+2 \sigma\right)^{-2}, \\
p_{\perp}=-\left(\frac{R}{2 \pi^{2} r}\right)\left(t^{2}+2 \sigma\right)^{-2}-\frac{R}{16 \pi^{2} r^{3}}\left(t^{2}+2 \sigma\right)^{-1}, \\
p_{r}=0-\frac{r R}{8 \pi^{2}(R-r) r^{3}}\left(t^{2}+2 \sigma\right)^{-1} .
\end{gathered}
$$

The vanishing leading term in Eq. (13) emphasizes that the radial pressure is in a sense less singular than the other components, which removes any contradiction with [10]. Very surprising, however, is the remaining term in 
Eq. (13), which displays a divergence at the surface, $r=R$. This divergence is purely geometrical, not ultraviolet; it exists although the cutoff $t$ is still finite! It constitutes a failure of the single-reflection optical approximation. (It arises in terms involving 2 derivatives of $D$, and those are of same order as terms omitted from $\bar{T} \approx D(t+2 \sigma)^{-1}$. This is a generic limitation of WKB-type methods.)

Attempts to construct an optical approximation of higher order in stationary phase (routine in curved manifolds without boundary ${ }^{5}$ ) break down at boundaries. (See [12] for the analogous problem with the heat kernel.) Such expansions can't naturally yield arctangent terms, which are almost certainly present, as we'll see presently. Instead, we shall turn to the full multiple-reflection expansion, integrating over paths whose reflection need not be specular.

First, however, consider the implications of the conservation law, $\nabla_{\mu} T^{\mu \nu}=0$. Under the symmetry conditions of our problem, ${ }^{10}$ one of its components is

$$
\frac{\partial p_{r}}{\partial r}+\frac{2 p_{r}}{r}-\frac{2 p_{\perp}}{r}=0 \Rightarrow p_{r}=\frac{2}{r^{2}} \int^{r} p_{\perp} r d r .
$$

Then Eq. (12) suggests that $p_{r}$ must contain a term

$$
-\frac{R^{3}}{\pi^{2} t^{3} r^{2}} \frac{4 R^{2}+3 t^{2}}{\left(4 R^{2}+t^{2}\right)^{2}} \tan ^{-1}\left(\frac{t}{2(R-r)}\right) \sim-\frac{1}{8 \pi R t^{3}}
$$

(for $r=R, t \ll R$ ). Of all the terms in the integral (14), this one seem uniquely capable of supplying the expected pressure - but is off by a factor $-\frac{1}{2}$, to which we'll return shortly.

Now we consider the first term in the multiple-scattering expansion of Balian and Bloch: ${ }^{13}$

$$
G\left(k, \mathbf{r}, \mathbf{r}^{\prime}\right)-G_{0}=-2 \int_{S} d S_{\mathbf{q}} \frac{\partial G_{0}(k, \mathbf{r}, \mathbf{q})}{\hat{n}_{\mathbf{q}}} G_{0}\left(k, \mathbf{q}, \mathbf{r}^{\prime}\right)+\cdots
$$

$\mathrm{Liu}^{14}$ observed that because

$$
\bar{T}\left(t, \mathbf{r}, \mathbf{r}^{\prime}\right)=\frac{2}{\pi} \int_{0}^{\infty} e^{-t k} \operatorname{Im} G\left(k, \mathbf{r}, \mathbf{r}^{\prime}\right) d k,
$$

it follows that

$$
\bar{T}-\bar{T}_{0}=\frac{1}{4 \pi^{3}} \int d S_{\mathbf{q}} \cos \theta_{1}\left(\frac{1}{r_{1}^{2}\left(L^{2}+t^{2}\right)}+\frac{2 L^{2}}{r_{1} r_{2}\left(L^{2}+t^{2}\right)^{2}}\right)+\cdots
$$

where $\cos \theta_{1} \equiv(\mathbf{q}-\mathbf{r}) \cdot \hat{n}_{\mathbf{q}}, r_{1} \equiv|\mathbf{r}-\mathbf{q}|, r_{2} \equiv\left|\mathbf{q}-\mathbf{r}^{\prime}\right|$, and $L \equiv r_{1}+r_{2}$. This formula applies to any surface, not just a sphere. (It can also be 
obtained directly from the single-scattering term in $\bar{T}$, in accordance with the philosophy of Sec. 2.)

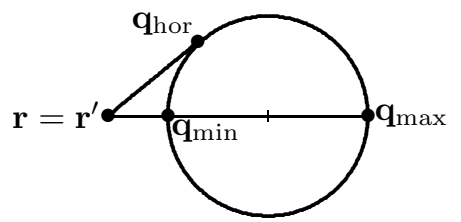

Now specialize to the (exterior of the) sphere. ${ }^{14}$ There are three critical lengths of paths,

$$
L_{\min }=2(r-R), \quad L_{\max }=2(r+R), \quad L_{\mathrm{hor}}=2 \sqrt{r^{2}-R^{2}} .
$$

Paths intersecting the sphere beyond the point of tangency at $L=L_{\mathrm{hor}}$ are expected to cancel approximately against paths with three reflections, at least in the optical limit. ${ }^{15}$

The integral from the visible region $\left(L_{\mathrm{min}}, L_{\mathrm{hor}}\right)$ is

$$
\frac{1}{4 \pi^{2} a}\left[\frac{2 R}{t^{2}+L_{\min }^{2}}+\frac{1}{t}\left(\tan ^{-1} \frac{L_{\mathrm{min}}}{t}-\tan ^{-1} \frac{L_{\mathrm{hor}}}{t}\right)\right] .
$$

The first term here is recognized as the optical approximation to $\bar{T}$. The second term is the predicted arctangent. (It gives a correction of order $O\left(L_{\min }^{-3}\right)$ to the leading term in $\rho(\mathbf{r}, 0)$, which is $O\left(L_{\min }^{-4}\right)$ with $L_{\min }=$ $2(r-R) \rightarrow 0$ at the boundary.)

The integral from the shadow region $\left(L_{\mathrm{hor}}, L_{\max }\right)$ is

$$
\frac{1}{4 \pi^{2} a}\left[\frac{2 R}{t^{2}+L_{\max }^{2}}+\frac{1}{t}\left(\tan ^{-1} \frac{L_{\mathrm{hor}}}{t}-\tan ^{-1} \frac{L_{\max }}{t}\right)\right] .
$$

The first term is the optical term from reflection on the far side of the sphere. Although one expects this term to be compensated by terms with more reflections, in the full multiple-reflection integral a consistent truncation at $L_{\text {hor }}$ is not possible, because contributions from $L \approx L_{\text {min }}$ are entangled with those from comparably short 3-reflection paths. Better calculational technology is needed to get a good handle on these low-frequency details.

To get $\left\langle T^{\mu \nu}\right\rangle$ we need derivatives of $\bar{T}$ at $\mathbf{r}^{\prime}=\mathbf{r}$. (Evaluating the integrals for $\bar{T}$ at $\mathbf{r}^{\prime} \neq \mathbf{r}$ appears hopeless.) After much Mathematica work we reproduce in the limit of small $t$

$$
E \sim-\frac{R^{2}}{2 t^{3}}+\frac{5}{192 \pi R}+O\left(t^{2} / R^{3}\right)
$$


(cf. Eq. (9), whose $O\left(R / t^{2}\right)$ term comes from two reflections) and

$$
p_{r} \sim-\frac{1}{8 \pi R t^{3}}+\frac{1}{64 \pi R^{3} t}+O\left(t^{0} / R^{4}\right)
$$

the (cut-off) radial pressure is finite at the boundary and originates in the subdominant arctangent term. (So there is no contradiction with [9].)

This is a qualitative resolution of the sphere pressure paradox - but only qualitative. We found $p_{r} \sim-\left(8 \pi R t^{3}\right)^{-1}$ but were expecting $p_{r} \sim$ $+\left(4 \pi R t^{3}\right)^{-1}$. This prompted us to look at flat plates intersecting at right angles in dimension 3 , where we find the same discrepancy $-\frac{1}{2}$ ! That in turn forced a reexamination of the 2-dimensional calculation in [1], mentioned above. It turns out that there the numerical factor is correct but the sign is wrong. (In dimension $n$ the discrepant factor (for flat plates) is $-1 /(n-1)$.)

\section{Paradox Future}

So, why is the energy-balance equation

$$
\frac{\partial E}{\partial h}=-\int_{S} p_{h}
$$

(for general parameter $h$ ) violated in the cutoff theory? (Note that a similar, nonparadoxical, result was found in [16].)

Let us take a critical look at the leading, bulk term in the vacuum $\left\langle T^{\mu \nu}(\mathbf{r})\right\rangle$, which we usually simply throw away. The ultraviolet cutoff yields

$$
T^{\mu \nu}=\frac{1}{2 \pi^{2} t^{4}} \operatorname{diag}(3,1,1,1) \equiv \frac{1}{2 \pi^{2} t^{4}} T_{\mathrm{rad}},
$$

proportional to the stress tensor of massless radiation. If we were to take it seriously, we would note that it is (i) ambiguous, (ii) not Lorentz-invariant, and (iii) inconsistent with Eq. (24).

As concerns ambiguity, note that elsewhere ${ }^{17}$ we argued that

$$
T^{\mu \nu} \propto \operatorname{diag}(-1,1,1,-3) \equiv T_{\text {Cas }},
$$

the tensor characteristic of conformally invariant Casimir energy ${ }^{18}$ (which happens to be invariant under Lorentz transformations parallel to the $x-$ $y$ plane). In that calculation the three space-time directions $(t, x, y)$ were treated alike, but differently from $z$. Effectively, we used a $k_{z}$ cutoff instead of a frequency cutoff.

This problem was confronted in the early years of quantum field theory in curved space-time. ${ }^{5,19-21}$ In particular, Christensen ${ }^{5}$ calculated the bulk vacuum stress tensor

$$
T^{\mu \nu}=\frac{1}{2 \pi^{2} t^{4}}\left(g^{\mu \nu}-4 u^{\mu} u^{\nu} \operatorname{sgn}\left(u_{\rho} u^{\rho}\right)\right),
$$


where $u^{\mu}$ was the unit 4-vector in the direction of "point separation". $T_{\text {rad }}$ corresponds to point-splitting in the time direction, $T_{\text {Cas }}$ to pointsplitting in the direction of a certain spatial coordinate. In the gravitational context it was taken as self-evident that a physically acceptable term of zeroth order in curvature must be covariant and hence proportional to

$$
T_{\text {dark }} \equiv \operatorname{diag}(-1,1,1,1)
$$

(i.e., to the metric tensor). It can then be absorbed by renormalization of the cosmological constant. Various rationalizations of this step appeared in the literature. Zel'dovich ${ }^{19}$ advocated Pauli-Villars regularization, which maintains Lorentz invariance at all times. Adler et al. and Christensen ${ }^{20,21}$ proposed to average over $u$ (getting 0 for massless fields). Others just "renormalized" direction-dependent terms, resolving any ambiguity by requiring $\nabla_{\mu} T^{\mu \nu}=0$ (conservation) for the remainder.

Unlike $T_{\text {rad }}$ and $T_{\text {Cas }}, T_{\text {dark }}$ satisfies Eq. (24). Note that the latter does not automatically follow from $\nabla_{\mu} T^{\mu \nu}=0$ for the material inside a container; it requires also an equation of state that is consistent with what actually happens to the "matter" when the volume of the container changes. For bulk vacuum energy, nothing happens, so only the dark-energy equation of state, $p=-\rho$, is allowed.

The lesson seems to be that a cutoff model is not a complete, consistent dynamical system, so formal properties such as covariance, conservation, and energy balance may be disrupted and need to be restored ad hoc.

In standard static Casimir problems, we have a rest frame, so this direction dependence has always been ignored. Now we see that the issue reemerges in the cutoff-dependent terms near boundaries. There is no apparent physical reason why point-splitting in the time direction should be preferred. Modeling of a real conductor suggests that a wave-number cutoff in directions parallel to the boundary may be more appropriate, ${ }^{4}$ and what is appropriate for the energy may not be so for the pressure. ${ }^{22}$

Numerically, the energy-balance equation (in particular, the sign) for rectangular boxes is disrupted because, for timelike point-splitting, the graph of the cut-off energy density displays a dip $(*)$ at the boundary that does not occur in the pressure function:

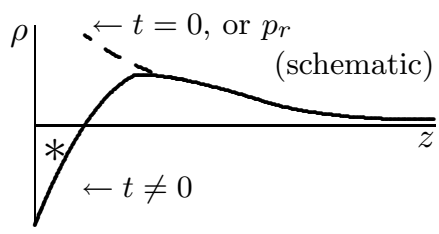


But it was precisely that dip that restored consistency with the integrated energy, resolving the first of the three paradoxes! Therefore, it may be that the famous formula

$$
E(t) \sim c_{0} V t^{-4}+c_{1} S t^{-3}+\cdots
$$

is totally meaningless as far as cutoff-dependent terms are concerned. In future work the full variety of possible cutoffs needs to be investigated in search of formal consistency of stress tensor components as well as physical plausibility in comparison with particular real boundary interactions.

\section{Acknowledgments}

This work was supported by National Science Foundation Grant PHY0554849. It incorporates input from Martin Schaden, my students Zhonghai Liu, Phuongmai Truong, and Kevin Resil, and the coauthors of [1].

\section{References}

1. S. A. Fulling, L. Kaplan, K. Kirsten, Z. H. Liu and K. A. Milton, J. Phys. A 42, 155402 (2009).

2. D. Deutsch and P. Candelas, Phys. Rev. D 20, 3063 (1979).

3. G. Barton, J. Phys. A 34, 4083 (2001).

4. G. Barton, J. Phys. A 37, 1011 (2004); ibid. 38, 2997 (2005).

5. S. M. Christensen, Phys. Rev. D 14, 2490 (1976).

6. F. H. Molzahn, T. A. Osborn and S. A. Fulling, Ann. Phys. 20464 (1990).

7. A. Scardicchio and R. L. Jaffe, Nucl. Phys. B 704, 552 (2005).

8. L. H. Ford and N. F. Svaiter, Phys. Rev. D 58, 065007 (1998).

9. A. Scardicchio and R. L. Jaffe, Nucl. Phys. B 743, 249 (2006).

10. D. Schwarz-Perlov and K. Olum, Phys. Rev. D 72, 065013 (2005).

11. K. A. Milton, Phys. Rev. D 68, 065020 (2003).

12. D. M. McAvity and H. Osborn, Class. Quantum Grav. 8, 603 (1991).

13. R. Balian and C. Bloch, Ann. Phys. 60, 401 (1970).

14. Z. H. Liu, Ph.D. dissertation, Texas A\&M University, 2009, http://www . math. tamu. edu/ fulling/qvac09/liudiss.pdf.

15. R. Balian and C. Bloch, Ann. Phys. 69, 76 (1972).

16. G. Barton, J. Phys. A 37, 3725 (2004).

17. K. A. Milton, The Casimir Effect, (World Scientific, Singapore, 2001); K. A. Milton, S. A. Fulling, P. Parashar, A. Romeo, K. V. Shajesh and J. A. Wagner, J. Phys. A 41, 164052 (2008).

18. L. S. Brown and G. J. Maclay, Phys. Rev. 184, 1272 (1969).

19. Ya. B. Zel'dovich, Sov. Phys. Usp. 11, 381 (1968).

20. S. L. Adler, J. Lieberman and Y. J. Ng, Ann. Phys. 106, 279 (1977).

21. S. M. Christensen, Phys. Rev. D 17, 946 (1978).

22. G. Barton, J. Phys. A 38, 3021 (2005). 